\title{
Processing of measures measured in underground polygonations
}

\author{
Larisa-Ofelia Filip $^{1 *}$, and Simona Cucaila ${ }^{2}$ \\ ${ }^{1}$ University of Petrosani, Faculty of Mining, 20 University Street, Petrosani, Romania \\ ${ }^{2}$ Ministry of Education, General Directorate for International Relations and Europeana Affairs, 28-30 \\ General H.M. Berthelot Street, Bucharest, Romania
}

\begin{abstract}
The information, which is the concrete basis for solving geodetic and topographic problems, comes from measurement observations made on quantities that are mainly angles and distances. The quality of the observations has an important role in achieving the objectives for which they are executed, in conditions of efficiency and safety. As topographically, underground works are conducted using polygonal paths, the methods used for processing measurements are of great interest.
\end{abstract}

The quality of the observations obtained from the measurements is a direct function of the volume of the observations and the accuracy of the instruments and techniques for their processing.

It is necessary, on the basis of the purpose for which the measurements are made, to establish the appropriate values in terms of size and accuracy, taking into account the economic aspect of the volume of necessary and sufficient observations required.

Considering the importance of underground polygons in the management of mining, hydrotechnical works, roads, etc., it is necessary to process the measured quantities (angles, distances) by rigorous methods based on the theory of small squares.

Underground polygons can be simple or networked [1]. However, as the networks are formed by simple polygonal routes, such a route will be analyzed (Figure 1).

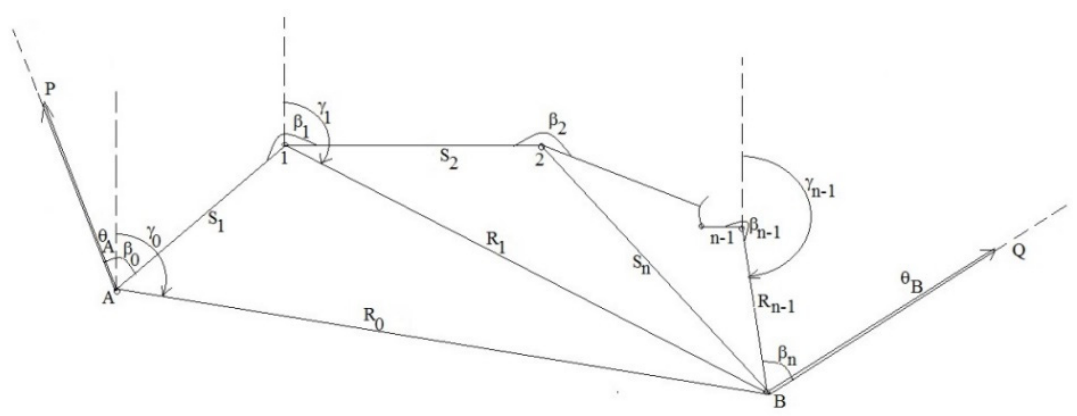

Fig. 1. Polygonal path.

*Corresponding author: larisafilip@yahoo.com 
The coordinates of points $\mathrm{A}, \mathrm{B}, \mathrm{P}, \mathrm{Q}$ are known as given quantities and angles $\beta$, respectively distances as natural quantities.

To determine the coordinates of the points $1,2, \ldots, n-1$, it is necessary and important to process the measured quantities in order to obtain their probable values.

For this purpose, it is observed that there are three quantities measured in addition to the required number, respectively the distance $S_{n}$ and angles $\beta_{n-1}, \beta_{n}$.

As a result, three geometric conditions must be met [2]:

- a condition of guidelines

- two coordinate conditions

The conditions are expressed by the equations:

$$
\begin{gathered}
\theta_{A}+[(\beta)] \pm n \cdot 200=\theta_{B} \\
x_{A}+[(\Delta x)]=x_{B} \\
y_{B}+[(\Delta y)]=y_{B}
\end{gathered}
$$

in which:

$(\beta)$ - the probable values of the angles

$(\Delta x),(\Delta y)$ - increases in coordinates set with probable values of angles and distances

If $v_{0}, v_{1}, v_{2}, \ldots, v_{n}, v_{S_{1}}, v_{S_{2}}, \ldots, v_{S_{n}}$ are the corrections of the measured angles and distances, the equations corresponding to the geometric conditions are:

$$
\begin{gathered}
v_{1}+v_{2}+\cdots+v_{n}+\omega_{1}=0 \\
v_{S_{1}} \cos \theta_{1}+v_{S_{2}} \cos \theta_{2}+\cdots+v_{S_{n}} \cos \theta_{n}-\frac{v_{0}}{\rho} R_{0} \sin \gamma_{0}-\frac{v_{1}}{\rho} R_{1} \sin \gamma_{1}-\cdots- \\
-\frac{v_{n-1}}{\rho} R_{n-1} \sin \gamma_{n-1}+\omega_{2}=0 \\
v_{S_{1}} \sin \theta_{1}+v_{S_{2}} \sin \theta_{2}+\cdots+v_{S_{n}} \sin \theta_{n}+\frac{v_{0}}{\rho} R_{0} \cos \gamma_{0}+\frac{v_{1}}{\rho} R_{1} \cos \gamma_{2}+\cdots+ \\
+\frac{v_{n-1}}{\rho} R_{n-1} \sin \gamma_{n-1}+\omega_{3}=0
\end{gathered}
$$

In the system (2) angle corrections with weights:

$$
p_{\beta_{0}}=p_{\beta_{1}}=\cdots=p_{\beta_{n}}=p
$$

and distances:

$$
p_{S_{1}} \neq p_{S_{2}} \neq \cdots \neq p_{S_{n}}
$$

It was noted with $\gamma_{0}, \gamma_{1}, \ldots, \gamma_{n-1}$ orientation distances $R_{0}, R_{1}, \ldots, R_{n-1}$.

The weights of the angles and sides can be considered equal if an artifice is used.

Divides the linear error $m_{S}$ to the angular error $m_{\beta}$ and the unit of measurement in which linear errors can be expressed results.

So if:

$$
m_{\beta}=4^{c c} \text { and } m_{S}=6 \mathrm{~cm}
$$


the unit of measurement is:

$$
e=\frac{6}{4}=1,5
$$

So:

$$
\left.\frac{m_{S}}{e}=\frac{6}{1,5}=4 \text { (units of } 1,5 \mathrm{~cm}\right)
$$

As a result, the measured distances are considered in units of measurement.

The equations in system (2) are solved on 2 groups of equations:

- group I - by equation

- group II - coordinate equations

For the first group, the system of normal equations is [3]:

$$
\begin{gathered}
{[a a] k_{1}+\omega_{1}=0 ;(n+1) k_{1}+\omega_{1}=0} \\
k_{1}=-\frac{\omega_{1}}{n+1}
\end{gathered}
$$

and corrections are obtained with the equalities:

$$
v_{i}^{\prime}=a_{i} k_{1} ; \quad v_{i}^{\prime}=-\frac{\omega_{1}}{n+1}
$$

For the second group, the coefficients must be transformed. Thus, the correction coefficients will be $\mathrm{B}$ and $\mathrm{C}$ obtained with the equalities:

$$
\begin{aligned}
B_{i} & =b_{i}+a_{i} \alpha_{1} \\
C_{i} & =c_{i}+a_{i} \alpha_{2}
\end{aligned}
$$

where $\alpha_{1}$ and $\alpha_{2}$ of the systems:

$$
\begin{aligned}
& {[a a] \alpha_{1}+[a b]=0} \\
& {[a a] \alpha_{2}+[a c]=0}
\end{aligned}
$$

But according to the scheme 1:

\begin{tabular}{|c|c|c|c|}
\hline Corection & $\mathrm{a}$ & $\mathrm{b}$ & $\mathrm{c}$ \\
\hline$v_{0}$ & 1 & $-\rho^{-1} R_{0} \sin \gamma_{0}$ & $\rho^{-1} R_{0} \cos \gamma_{0}$ \\
\hline$v_{1}$ & 1 & $-\rho^{-1} R_{1} \sin \gamma_{1}$ & $\rho^{-1} R_{0} \cos \gamma_{1}$ \\
\hline------- & ----------- & ------ \\
\hline$v_{n-1}$ & 1 & $---\rho^{-1} R_{n-1} \sin \gamma_{n-1}$ & $\rho^{-1} R_{n-1} \cos \gamma_{n-1}$ \\
\hline$v_{n}$ & 1 & - & - \\
\hline$v_{S_{1}}$ & 0 & $\cos \theta_{1}$ & $\sin \theta_{1}$ \\
\hline$v_{S_{2}}$ & 0 & $\cos \theta_{2}$ & $\sin \theta_{2}$ \\
\hline------- & -------------- & $\sin \theta_{n}$ \\
\hline$v_{S_{n}}$ & 0 & $\cos \theta_{n}$ & \\
\hline
\end{tabular}

$$
[a a]=n+1
$$




$$
\begin{aligned}
& {[a b]=-\frac{1}{\rho}[R \sin \gamma]} \\
& {[a c]=\frac{1}{\rho}[R \cos \gamma]}
\end{aligned}
$$

and:

$$
\begin{aligned}
\alpha_{1} & =\frac{1}{\rho(n+1)}[R \sin \gamma] \\
\alpha_{2} & =-\frac{1}{\rho(n+1)}[R \cos \gamma]
\end{aligned}
$$

With these we get:

$$
\begin{gathered}
B_{i}=-\frac{R_{i}}{\rho^{\prime \prime}} \sin \gamma_{i}+\frac{[R \sin \gamma]}{\rho^{\prime \prime}(n+1)} \\
B_{j}=\cos \theta_{j}+\frac{[R \sin \gamma]}{\rho^{\prime \prime}(n+1)} \\
i=0,1,2, \ldots, n-1 \\
j=1,2, \ldots, n \\
C_{i}=-\frac{R_{i}}{\rho^{\prime \prime}} \cos \gamma_{i}-\frac{[R \cos \gamma]}{\rho^{\prime \prime}(n+1)} \\
C_{j}=\sin \theta_{j}-\frac{[R \cos \gamma]}{\rho^{\prime \prime}(n+1)}
\end{gathered}
$$

The system of normal equations for the system of error equations of group II is [3]:

$$
\begin{aligned}
{[B B] K_{2}+[B C] K_{3}+\omega_{2} } & =0 \\
{[B C] K_{2}+[C C] K_{3}+\omega_{3} } & =0
\end{aligned}
$$

By solving the system(10) the correlates are obtained $K_{2}, K_{3}$ that:

$$
v^{\prime \prime}{ }_{i, j}=B_{i, j} K_{2}+C_{i, j} K_{3}
$$

Total corrections are obtained:

$$
v_{i, j}=v_{i}^{\prime}+v_{i, j}^{\prime \prime}
$$

In topographic practice, there are frequent cases when the angles of the polygons are measured with high precision. This is achieved by small non-concluding on the orientations and consequently small corrections of the angles.

In such a situation the measured angles are considered with the same precision, and the non-closing on the orientations is distributed in equal parts on all the measured angles.

With the angles thus corrected (compensated) the orientations of the sides are calculated and together with them the coordinates of the points of the polygon.

It turns out that only the sides can be further processed compensated.

There will be only two conditions for which there are two equations of form: 


$$
\begin{aligned}
& v_{S_{1}} \cos \theta_{1}+v_{S_{2}} \cos \theta_{2}+\cdots+v_{S_{n}} \cos \theta_{n}+w_{x}=0 \\
& v_{S_{1}} \sin \theta_{1}+v_{S_{2}} \sin \theta_{2}+\cdots+v_{S_{n}} \sin \theta_{n}+w_{y}=0
\end{aligned}
$$

Weights are introduced for sides of different lengths, but we consider the above artifice and consequently in the following calculations we do not use weights. For the system (13) of normal equations is:

$$
\begin{array}{r}
{\left[\cos ^{2} \theta\right] K_{1}+[\sin \theta \cos \theta] K_{2}+w_{x}=0} \\
{[\sin \theta \cos \theta] K_{1}+\left[\sin ^{2} \theta\right] K_{2}+w_{y}=0}
\end{array}
$$

By solving the system (14) you get:

$$
\begin{aligned}
K_{1} & =\frac{[\sin \theta \cos \theta] w_{y}-\left[\sin ^{2} \theta\right] w_{x}}{\left[\sin ^{2} \theta\right]\left[\cos ^{2} \theta\right]-[\sin \theta \cos \theta]^{2}} \\
K_{2} & =\frac{[\sin \theta \cos \theta] w_{x}-\left[\cos ^{2} \theta\right] w_{y}}{\left[\sin ^{2} \theta\right]\left[\cos ^{2} \theta\right]-[\sin \theta \cos \theta]^{2}}
\end{aligned}
$$

Length corrections, according to the theory of conditional measurements are obtained with equalities:

$$
\begin{gathered}
v_{S_{i}}=a_{i} K_{1}+b_{i} K_{2} \\
i=1,2, \ldots, n
\end{gathered}
$$

In practice there is often a particular case regarding the geometric shape of the polygonal path [4]. There are polygonal paths developed in the AB direction with the measured angles close to $200^{g}$. For such routes the orientations of the sides and direction $R_{i}$ they are equal.

So:

$$
\begin{gathered}
\theta_{1}=\theta_{2}=\cdots=\theta_{n}=\theta \\
\gamma_{0}=\gamma_{1}=\cdots=\gamma_{n-1}=\gamma
\end{gathered}
$$

And the system of error equations has the form:

$$
\begin{gathered}
v_{0}+v_{1}+v_{2}+\cdots+v_{n}+\omega_{1}=0 \\
\cos \theta\left(v_{S_{1}}+v_{S_{2}}+\cdots+v_{S_{n}}\right)-\sin \theta\left(R_{0} v_{0}+R_{1} v_{1}+\cdots+R_{n-1} v_{n-1}\right)+\omega_{2}=0 \\
\sin \theta\left(v_{S_{1}}+v_{S_{2}}+\cdots+v_{S_{n}}\right)+\cos \theta\left(R_{0} v_{0}+R_{1} v_{1}+\cdots+R_{n-1} v_{n-1}\right)+\omega_{3}=0
\end{gathered}
$$

Equations 2 and 3 in the system (17) are multiplied with $\sin \theta$ and $\cos \theta$ după which decreases and results:

$$
R_{0} v_{0}+R_{1} v_{1}+\cdots+R_{n-1} v_{n-1}+\omega_{3} \cos \theta-\omega_{2} \sin \theta=0
$$

The mentioned equations are multiplied by $\cos \theta$ and $\sin \theta$ after which by assembly it results:

$$
v_{S_{1}}+v_{S_{2}}+\cdots+v_{S_{n}}+\omega_{2} \cos \theta+\omega_{3} \sin \theta=0
$$


As a result, the system of error equations has the form:

$$
\begin{gathered}
v_{0}+v_{1}+v_{2}+\cdots+v_{n}+\omega_{1}=0 \\
R_{0} v_{0}+R_{1} v_{1}+\cdots+R_{n-1} v_{n-1}+\omega_{3} \cos \theta-\omega_{2} \sin \theta=0 \\
v_{S_{1}}+v_{S_{2}}+\cdots+v_{S_{n}}+\omega_{2} \cos \theta+\omega_{3} \sin \theta=0
\end{gathered}
$$

Analyzing the system of error equations (20) it is found that in the first two equations there are errors of angles and in equation 3 errors of sides. The conclusion is that in polygons made in one direction, the errors of lengths act distinctly from the errors of angles.

Moreover, in the system of normal equations the correlation coefficients $[a c]$ and $[b c]$ are equal to zero.

It is also found that the terms free $w_{2}$ and $w_{3}$ are obtained with relationships:

$$
\begin{aligned}
& w_{2}=w_{y} \cos \theta-w_{x} \sin \theta \\
& w_{3}=w_{y} \sin \theta+w_{x} \cos \theta_{0}
\end{aligned}
$$

which expresses their geometric meaning, respectively the influence of the measured quantities in determining the transverse and longitudinal errors in point B.

If group solving is used, the transformed coefficients are:

$$
\begin{aligned}
& B_{i}=-\frac{R_{i}}{\rho} \sin \theta+\frac{[R]}{\rho(n+1)} \sin \theta=\left(\frac{[R]}{\rho(n+1)}-\frac{R_{i}}{\rho}\right) \sin \theta \\
& B_{j}=\cos \theta+\frac{[R]}{\rho(n+1)} \sin \theta=\left(1+\frac{[R]}{\rho(n+1)} \operatorname{tg} \theta\right) \cos \theta \\
& C_{i}=\frac{R_{i}}{\rho} \sin \theta-\frac{[R]}{\rho(n+1)} \cos \theta=\left(\frac{R_{i}}{\rho}-\frac{[R]}{\rho(n+1)}\right) \cos \theta \\
& C_{j}=\sin \theta-\frac{[R]}{\rho(n+1)} \cos \theta=\left(1-\frac{[R]}{\rho(n+1)} \operatorname{ctg} \theta\right) \sin \theta
\end{aligned}
$$

Note:

$$
\frac{[R]}{\rho(n+1)}=K-\text { it can be easily obtained }
$$

and then:

$$
\begin{gathered}
B_{i}=\left(K-\frac{R_{i}}{\rho}\right) \\
B_{j}=(1+K \operatorname{tg} \theta) \cos \theta
\end{gathered}
$$




$$
\begin{gathered}
C_{i}=\left(\frac{R_{i}}{\rho}-K\right) \cos \theta \\
C_{j}=(1-K \operatorname{ctg} \theta) \sin \theta
\end{gathered}
$$

By analyzing the polygonal paths performed on the processing of the measured quantities, methods are established that have the role of contributing to the increase of safety in the execution of the underground objectives.

\section{References}

1. N. Dima, ș.a., Mining topography (Corvin, Deva, 1996)

2. N. Dima, Geodesy (Universitas, Petroșani, 2005)

3. N. Dima, O. Herbei, I. Vereș, Error theory and least squares method (Universitas, Petroșani, 1999)

4. $\quad$ L. Filip, N. Dima, Special mining topography works (Universitas, Petroșani, 2014) 